PROCEEDINGS OF THE

AMERICAN MATHEMATICAL SOCIETY

Volume 127, Number 9, Pages 2783-2791

S 0002-9939(99)04853-4

Article electronically published on April 15, 1999

\title{
A NON-METRIZABLE COMPACT LINEARLY ORDERED TOPOLOGICAL SPACE, EVERY SUBSPACE OF WHICH HAS A $\sigma$-MINIMAL BASE
}

\author{
WEI-XUE SHI
}

(Communicated by Alan Dow)

\begin{abstract}
A collection $\mathcal{D}$ of subsets of a space is minimal if each element of $\mathcal{D}$ contains a point which is not contained in any other element of $\mathcal{D}$. A base of a topological space is $\sigma$-minimal if it can be written as a union of countably many minimal collections. We will construct a compact linearly ordered space $X$ satisfying that $X$ is not metrizable and every subspace of $X$ has a $\sigma$-minimal base for its relative topology. This answers a problem of Bennett and Lutzer in the negative.
\end{abstract}

\section{INTRODUCTION}

The concept of $\sigma$-minimal bases was introduced by Aull in [1] and it was pointed out that every quasi-developable space has a $\sigma$-minimal base. Bennett and others proved that a space $X$ with a $\sigma$-minimal base need not be quasi-developable even if every subspace of $X$ has a $\sigma$-minimal base (cf. [2] and [3]). On the other hand, the condition compactness forces a quasi-developable space to be metrizable, but a compact space with a $\sigma$-minimal base need not be metrizable even if the space is a linearly ordered topological space (LOTS) [3]. It is observed that the space constructed in [3] has a subspace which has no $\sigma$-minimal base. Recently Bennett and Lutzer constructed a non-metrizable LOTS such that every subspace of it has a $\sigma$-minimal base for its relative topology, but the LOTS is not itself compact [5]. So the following question posed by Bennett and Lutzer (cf. [3], [4], [6] and [9]) becomes more interesting.

Problem 1. Suppose that $X$ is a compact linearly ordered topological space and suppose that every subspace of $X$ has a $\sigma$-minimal base for its relative topology. Must $X$ be metrizable?

In this paper, we will answer this problem negatively by constructing a nonmetrizable compact LOTS $X$ such that every subspace of $X$ has a $\sigma$-minimal base for its relative topology.

Received by the editors October 25, 1996 and, in revised form, November 15, 1997.

1991 Mathematics Subject Classification. Primary 54F05, 54G20, 54E35.

Key words and phrases. $\sigma$-minimal base, metrizable, linearly ordered topological space, special Aronszajn tree, quasi-developable. 
Recall that a LOTS (a linearly ordered topological space) is a triple $\langle X, \lambda, \leqslant\rangle$, where $\langle X, \leqslant\rangle$ is an ordered set and $\lambda$ is the interval topology on $\langle X, \leqslant\rangle$ and a GO-space (a generalized ordered space) is a triple $\langle X, \tau \leqslant\rangle$, where $\tau$ is a topology on $\langle X, \leqslant\rangle$ which is $T_{1}$ and has a local base consisting of ordered convex sets at every point of $X$. A collection $\mathcal{D}$ of subsets of a space is said to be minimal if for every proper subcollection $\mathcal{D}^{\prime}$ of $\mathcal{D}, \cup \mathcal{D}^{\prime} \varsubsetneqq \bigcup \mathcal{D}$. A base $\mathcal{B}$ of a space is called a $\sigma$-minimal base if $\mathcal{B}=\bigcup\left\{\mathcal{B}_{n} \mid n \in \omega_{0}\right\}$, where for each $n \in \omega_{0}, \mathcal{B}_{n}$ is minimal.

We use an Aronszajn tree to construct our LOTS satisfying the required conditions. Now we review some related definitions and results.

A tree is a partially ordered set $\left\langle T, \leqslant_{T}\right\rangle$, simply written as $T$, such that for every $t \in T$ the set $(\cdot, t)_{T}=\left\{s \in T \mid s<_{T} t\right\}$ is well-ordered. The height $\mathrm{ht}_{T}(t)$ of $t$ in $\left\langle T, \leqslant_{T}\right\rangle$ is the order type of $(\cdot, t)_{T}$. The $\alpha$ th level of $T$ is the set $T_{\alpha}=\{t \in$ $\left.T \mid h_{T}(t)=\alpha\right\}$. The height $\operatorname{ht}(T)$ of $T$ is the ordinal $\min \left\{\alpha \mid T_{\alpha}=\emptyset\right\}$. A chain of a tree $T$ is a totally ordered subset of $T$. A branch of a tree $T$ is a maximal chain of $T$. If $x$ is a branch of a tree, then we denote the order type of $x$ by bht $(x)$. An antichain of a tree $T$ is a set of pairwise incomparable points of $T$. A path $p$ of $T$ is a chain such that for each $t \in p,(\cdot, t)_{T} \subset p$. We use ht $(p)$ to denote the order type of a path $p$. A node of a tree $T$ is any equivalence class of the relation $\sim$ defined on $T$ by $s \sim t$ if and only if $(\cdot, s)_{T}=(\cdot, t)_{T}$. Obviously each level is an antichain and a disjoint union of nodes. Especially $T_{0}$ is a node since for any $t, s \in T_{0},(\cdot, s)_{T}=(\cdot, t)_{T}=\emptyset$.

Let $T$ be a tree and let $\mathcal{N}(T)$ be the set of all nodes of $T$. If $p$ is a bounded path of $T$, let $N_{p}$ be the first level of the tree

$$
\left\{t \in T \mid s<_{T} t \text { for every } s \in p\right\} \text {. }
$$

Then $N_{p} \in \mathcal{N}(T)$.

Let $B_{T}$ be the set of all branches of $T$. Suppose that each $N \in \mathcal{N}(T)$ is endowed with a linear ordering $\leqslant_{N}$. Then the lexicographical ordering $\preccurlyeq$ on $B_{T}$ induced by $\left\{\leqslant_{N} \mid N \in \mathcal{N}(T)\right\}$ is defined by

$$
l \preccurlyeq m \text { if and only if } l_{N} \leqslant{ }_{N} m_{N},
$$

where $N=N_{l \cap m},\left\{l_{N}\right\}=l \cap N$ and $\left\{m_{N}\right\}=m \cap N$. Then $\preccurlyeq$ is a linear ordering on $B_{T}$. For every $t \in T$, let $B_{t}=\left\{m \in B_{T} \mid t \in m\right\}$. Then it is easy to see that $B_{t}$ is a convex set in $\left\langle B_{T}, \preccurlyeq\right\rangle$.

It is known that, if $\left\langle N, \leqslant_{N}\right\rangle$ is a complete linear ordering for each $N \in \mathcal{N}(T)$, then $\left\langle B_{T}, \preccurlyeq\right\rangle$ is also complete (see [10, Proposition 2.5]). For an ordinal $\alpha$, by $\alpha^{+}$ we mean the successor of $\alpha$, for a subset $Y$ of $B_{T}$, let $Y\lceil\alpha$ denote the set $\{x \in$ $Y \mid \operatorname{bht}(x)<\alpha\}$, and for an $x \in B_{T}$, let $x\left\lceil\alpha\right.$ denote the set $\left\{t \in x \mid h_{T}(t)<\alpha\right\}$. A tree $T$ is called an Aronszajn tree if ht $(T)=\omega_{1}$ and each branch and each level of it are countable. A tree $T$ is said to be special if $T$ is the union of countably many antichains. It is well-known that there is a special Aronszajn tree in ZFC (see [10, Theorem 5.2]).

For a space $X$ and its subset $Y$, the interior and closure of $Y$ in $X$ are denoted by $\operatorname{int}_{X} Y$ and $\operatorname{cl}_{X} Y$ respectively. For an ordered space $X$ and a subspace $Y$ of $X$, if $a, b \in Y$ and $a<b$, by $(a, b)_{Y}$ we mean the open interval taken in $Y$. Define the intervals $[a, b]_{Y},(a, b]_{Y},[a, b)_{Y}$ analogously. For undefined terminology we refer to $[7]$ and $[10]$. 


\section{A CONSTRUCtion of A LOTS AND SOME LEMmas}

Let $T$ be the Aronszajn tree constructed as in [10, Theorem 5.2]. $T$ is special since $T$ is $\mathbb{Q}$-embeddable. Thus $T=\bigcup\left\{A_{n} \mid n \in \omega_{0}\right\}$, where each $A_{n}$ is an antichain of $T$. From the construction of $T$ it is easy to see that the following facts are true.

Fact 1. For each $N \in \mathcal{N}(T)$ with $N \subset T_{\alpha},|N|=\omega_{0}$ if $\alpha$ is a successor ordinal or $\alpha=0$, and $|N|=1$ if $\alpha$ is a limit ordinal and $\alpha \neq 0$.

Fact 2. No branch of $T$ has a maximum element.

Suppose $N \in \mathcal{N}(T)$. If $N \subset T_{\alpha}$ for some successor ordinal $\alpha$ or $\alpha=0$, define a linear ordering $\leqslant_{N}$ on $N$ such that $\leqslant_{N}$ well-orders $N$ and $\left\langle N, \leqslant_{N}\right\rangle$ has the order type $\omega_{0}+1$. Then we may write $N=\left\{a(N)_{n} \mid n \in \omega_{0}+1\right\}$ such that

$$
a(N)_{0}<_{N} a(N)_{1}<_{N} \ldots<_{N} a(N)_{n}<_{N} \ldots<_{N} a(N)_{\omega_{0}} .
$$

If $N \subset T_{\alpha}$ for some limit ordinal $\alpha>0$, let $\leqslant_{N}$ be the trivial ordering on $N$. Then for every $N \in \mathcal{N}(T),\left\langle N, \leqslant_{N}\right\rangle$ is complete. Let $\preccurlyeq$ be the lexicographical ordering on $B_{T}$ induced by $\left\{\leqslant_{N} \mid N \in \mathcal{N}(T)\right\}$ and let $\lambda$ be the interval topology on $\left\langle B_{T}, \preccurlyeq\right\rangle$. Then $\left\langle B_{T}, \lambda, \preccurlyeq\right\rangle$ is a compact LOTS since it is complete and has maximum and minimum points. We will simply denote $\left\langle B_{T}, \lambda, \preccurlyeq\right\rangle$ by $B_{T}$. For an $x \in B_{T}$, by Fact $2, \operatorname{bht}(x)$ is a limit ordinal. For $\alpha<\operatorname{bht}(x)$, let $N(x, \alpha)=N_{x \nmid \alpha}$. Put

$$
\begin{aligned}
& B_{T 0}=\left\{x \in B_{T} \mid \text { there is } \beta<\operatorname{bht}(x) \text { such that, for each } \alpha\right. \\
& \left.\quad \text { with } \beta<\alpha^{+}<\operatorname{bht}(x), x \cap N\left(x, \alpha^{+}\right)=\left\{a\left(N\left(x, \alpha^{+}\right)\right)_{0}\right\}\right\}, \\
& B_{T 1}=\left\{x \in B_{T} \mid \text { there is } \beta<\operatorname{bht}(x) \text { such that, for each } \alpha\right. \\
& \left.\quad \text { with } \beta<\alpha^{+}<\operatorname{bht}(x), x \cap N\left(x, \alpha^{+}\right)=\left\{a\left(N\left(x, \alpha^{+}\right)\right)_{\omega_{0}}\right\}\right\},
\end{aligned}
$$

and

$$
B_{T 2}=B_{T}-\left(B_{T 1} \cup B_{T 0}\right) .
$$

That is, each branch $x \in B_{T 1}$ always picks up the maximum point in each node which $x$ meets at levels $>\beta$ and each branch $x \in B_{T 0}$ always picks up the minimum point in each node which $x$ meets at levels $>\beta$. For $x \in B_{T i}, i=0,1$, let

$$
\begin{aligned}
& \eta_{x}=\min \{\beta<\operatorname{bht}(x) \mid \text { for each } \alpha \text { with } \\
& \left.\qquad \beta<\alpha^{+}<\operatorname{bht}(x), x \cap N\left(x, \alpha^{+}\right)=\left\{a\left(N\left(x, \alpha^{+}\right)\right)_{\rho(i)}\right\}\right\}
\end{aligned}
$$

where $\rho(i)= \begin{cases}0, & \text { if } i=0, \\ \omega_{0}, & \text { if } i=1,\end{cases}$

and put

$$
\begin{aligned}
& B_{T i}^{s u c}=\left\{x \in B_{T i} \mid \eta_{x} \text { is a successor ordinal or } \eta_{x}=0\right\}, \\
& B_{T i}^{l i m}=\left\{x \in B_{T i} \mid \eta_{x} \text { is a limit ordinal and } \eta_{x} \neq 0\right\},
\end{aligned}
$$

and

$$
B_{T 0}^{\text {suc } 1}=\left\{x \in B_{T 0}^{s u c} \mid x \cap N\left(x, \eta_{x}\right)=\left\{a\left(N\left(x, \eta_{x}\right)\right)_{\omega_{0}}\right\}\right\} .
$$


Thus each branch $x \in B_{T 0}^{\text {suc1 }}$ picks up the maximum point in the node which $x$ meets at the $\eta_{x}$ th level and always picks up the minimum point in the nodes which $x$ meets above the $\eta_{x}$ th level.

Since $\operatorname{ht}(T)=\omega_{1}$, it is easy to see the following fact.

Fact 3. For $i=0,1,\left|B_{T i}\right|=\omega_{1}$ and if $\alpha<\omega_{1} \mid B_{T i}\left\lceil\alpha \mid=\omega_{0}\right.$.

Suppose $t \in T_{\alpha}$. By the definition of the ordering $\preccurlyeq$, the point $x$ in $B_{t} \cap B_{T 1}$ with $\eta_{x} \leqslant \alpha$ is the maximum point of $B_{t}$ and the point $x$ in $B_{t} \cap B_{T 0}$ with $\eta_{x} \leqslant \alpha$ is the minimum point of $B_{t}$. So we have

Fact 4. For each $t \in T, B_{t}$ is a closed interval in $B_{T}$, the maximum point of $B_{t}$ is in $B_{T 1}$ and the minimum point of $B_{t}$ is in $B_{T 0}$.

The following is an extension of the proof of Theorem 5.1 in [10].

Lemma 1. $\left\langle B_{T}, \lambda, \preccurlyeq\right\rangle$ is a first countable space.

Proof. Suppose that $B_{T}$ is not first countable. Then there is an $x \in B_{T}$ such that $x$ has no countable neighborhood base. So there is an increasing sequence $\left\{a_{\alpha} \mid \alpha \in\right.$ $\left.\omega_{1}\right\}$ homeomorphic to $\omega_{1}$ or decreasing sequence $\left\{b_{\alpha} \mid \alpha \in \omega_{1}\right\}$ homeomorphic to the converse of $\omega_{1}$ in $B_{T}$. For instance, there is an increasing sequence $A=\left\{a_{\alpha} \mid \alpha \in\right.$ $\left.\omega_{1}\right\}$. Then for each $\alpha \in \omega_{1}$, there is a $t_{\alpha} \in T_{\alpha}$ such that $\left\{a \in A \mid t_{\alpha} \in a\right\}$ is uncountable since $T_{\alpha}$ is countable. If for $\alpha<\beta<\omega_{1}, t_{\alpha} \nless_{T} t_{\beta}$, then $t_{\alpha}$ and $t_{\beta}$ are incomparable. Hence $B_{t_{\alpha}} \cap B_{t_{\beta}}=\emptyset$. Then both $B_{t_{\alpha}} \cap A$ and $B_{t_{\beta}} \cap A$ are uncountable subsequences of $A$. Since $B_{t_{\alpha}}$ and $B_{t_{\beta}}$ are disjoint convex sets in $B_{T}$, $A$ cannot be increasing, a contradiction. Thus $\left\{t_{\alpha} \mid \alpha \in \omega_{1}\right\}$ is an uncountable chain in $T$. This is impossible because $T$ is an Aronszajn tree.

Lemma 2. Let $X$ be a subspace of $\left\langle B_{T}, \lambda, \preccurlyeq\right\rangle$. The following conditions are equivalent.

(1) $X$ is separable.

(2) $\{\operatorname{bht}(x) \mid x \in X\}$ is not cofinal in $\omega_{1}$.

(3) There is a countable collection $\mathcal{P}$ of open sets in $\left\langle B_{T}, \lambda, \preccurlyeq\right\rangle$ such that for any $x \in X$ and any open neighborhood $U$ of $x$ in $B_{T}$, there is $a V \in \mathcal{P}$ such that $x \in V \subset U$.

(4) $X$ has a countable base.

Proof. (1) $\Rightarrow(2)$. Let $Y$ be a countable dense subset of $X$. Then $\{\operatorname{bht}(x) \mid x \in Y\}$ has an upper bound $\alpha$, and clearly we may take $\alpha$ as a limit ordinal. So $Y \subset B_{T}\left\lceil\alpha^{+}\right.$. Notice that $B_{T}-B_{T}\left\lceil\alpha^{+}=\bigcup\left\{B_{t} \mid t \in T_{\alpha}\right\}\right.$ and each $B_{t}$ is a closed interval in $B_{T}$. Hence among the elements of $B_{T}$ with the order type large than $\alpha$, only the endpoints of $B_{t}$ 's where $t \in T_{\alpha}$ possibly belong to $X \subset \operatorname{cl}_{B_{T}} Y$. The set of all endpoints of $B_{t}$ 's with $t \in T_{\alpha}$ is countable since $\left|T_{\alpha}\right|=\omega_{0}$. It follows that $\left\{\operatorname{bht}(x) \mid x \in X \subset \operatorname{cl}_{B_{T}} Y\right\}$ is not cofinal in $\omega_{1}$.

$(2) \Rightarrow(3)$. Let $\alpha$ be an upper bound of $\{\operatorname{bht}(x) \mid x \in X\}$ and assume that $\alpha$ is a limit ordinal. Then $X \subset B_{T}\left\lceil\alpha^{+}\right.$. The topology on $\operatorname{cl}_{B_{T}}\left(B_{T}\left\lceil\alpha^{+}\right)\right.$as a subspace of $B_{T}$ coincides with the topology on $\mathrm{cl}_{B_{T}}\left(B_{T}\left\lceil\alpha^{+}\right)\right.$as a LOTS since $\operatorname{cl}_{B_{T}}\left(B_{T}\left\lceil\alpha^{+}\right)\right.$is compact as a closed subspace of $B_{T}$. Let $\mathcal{I}$ be the collection of the convex components of $B_{T}-\operatorname{cl}_{B_{T}}\left(B_{T}\left\lceil\alpha^{+}\right)\right.$. Then $|\mathcal{I}| \leqslant \omega_{0}$ since each element $I$ of $\mathcal{I}$ contains an element of $\left\{\operatorname{int}_{B_{T}} B_{t} \mid t \in T_{\alpha}\right\}$ and $\left|T_{\alpha}\right|=\omega_{0}$. Let $T^{*}=$ $\bigcup\left\{x \mid x \in B_{T}\left\{\alpha^{+}\right\}=\bigcup\left\{T_{\beta} \mid \beta<\alpha\right\}\right.$. It follows from the definition of $T$ that for each $t \in T^{*}, \mid B_{t}\left\lceil\alpha^{+} \mid>\omega_{0}\right.$. Hence $\operatorname{int}_{B_{T}} B_{t} \cap B_{T}\left\lceil\alpha^{+} \neq \emptyset\right.$. Fix a point 
$x_{t} \in \operatorname{int}_{B_{T}} B_{t} \cap B_{T}\left\lceil\alpha^{+}\right.$and let $Y=\left\{x_{t} \mid t \in T^{*}\right\}$. Then $|Y|=\omega_{0}$ since $\left|T^{*}\right|=$ $\omega_{0}$. For any $x \in B_{T}\left\lceil\alpha^{+}\right.$and a convex neighborhood $\left(x_{1}, x_{2}\right)_{B_{T}}$ of $x$ in $B_{T}$, let $\beta=\max \left\{\operatorname{ht}\left(x_{1} \cap x\right)\right.$, ht $\left.\left(x \cap x_{2}\right)\right\}$ and $\{t\}=x \cap N\left(x, \beta^{+}\right)$. Then $t \in T^{*}$ and $B_{t} \subset\left(x_{1}, x_{2}\right)_{B_{T}}$. Hence $\left(x_{1}, x_{2}\right)_{B_{T}} \cap Y \neq \emptyset$. Therefore $Y$ is a dense subset of $\operatorname{cl}_{B_{T}}\left(B_{T}\left\lceil\alpha^{+}\right)\right.$. Recall that a point of a linearly ordered set is a jump point if the point has an immediate successor. It is easy to show that if $x \in B_{T}\left\lceil\alpha^{+} \cap B_{T 2}\right.$, then for any $y \in B_{T}$ with $x \prec y,(x, y)_{B_{T}} \cap B_{T}\left\lceil\alpha^{+} \neq \emptyset\right.$. So no element of $B_{T}\left\lceil\alpha^{+} \cap B_{T 2}\right.$ is a jump point of $\mathrm{cl}_{B_{T}}\left(B_{T}\left\lceil\alpha^{+}\right)\right.$. Notice that

$$
\operatorname{cl}_{B_{T}}\left(B_{T}\left\lceil\alpha^{+}\right)-B_{T}\left\lceil\alpha^{+} \subset\left\{e(t) \mid e(t) \text { is an endpoint of } B_{t}, \quad t \in T_{\alpha}\right\} .\right.\right.
$$

Therefore the set of all jump points of $\operatorname{cl}_{B_{T}}\left(B_{T}\left\lceil\alpha^{+}\right)\right.$is a subset of the following countable set

$$
\left(B_{T 0} \cup B_{T 1}\right) \mid \alpha^{+} \cup\left\{e(t) \mid e(t) \text { is an endpoint of } B_{t}, \quad t \in T_{\alpha}\right\} .
$$

It is known that a LOTS has a countable base if the LOTS is separable and the set of its jump points is countable (see the insert of [8]). So $\mathrm{cl}_{B_{T}}\left(B_{T}\left\lceil\alpha^{+}\right)\right.$has a countable base $\mathcal{C}$ consisting of open intervals in $\operatorname{cl}_{B_{T}}\left(B_{T}\left\lceil\alpha^{+}\right)\right.$. By Lemma 1 , $B_{T}$ is first countable. For each endpoint $x$ of $I \in \mathcal{I}$, let $\mathcal{V}(x)$ be the countable neighborhood base at $x$ in $B_{T}$. For each $C \in \mathcal{C}$, let $J_{C}$ be the open interval in $B_{T}$ having the same endpoints with $C$. Put

$$
\mathcal{P}=\left\{J_{C} \mid C \in \mathcal{C}\right\} \cup(\bigcup\{\mathcal{V}(x) \mid x \text { is an endpoint of } I, I \in \mathcal{I}\}) .
$$

Then $\mathcal{P}$ is a countable collection.

We prove that $\mathcal{P}$ is the required collection. Take any $x \in X$ and any open neighborhood $U$ of $x$ in $B_{T}$. If $x$ is an endpoint of $I \in \mathcal{I}$, an element $V \in \mathcal{V}(x) \subset \mathcal{P}$ is contained in $U$. Next suppose that $x$ is not an endpoint of $I$ for any $I \in \mathcal{I}$ and $U$ is a neighborhood of $x$ in $B_{T}$. We may assume that $U=\left(u_{0}, u_{1}\right)_{B_{T}}$. If $u_{0}$ (or $\left.u_{1}\right)$ is not in $\operatorname{cl}_{B_{T}}\left(B_{T}\left\lceil\alpha^{+}\right)\right.$, then $u_{0}$ (or $\left.u_{1}\right)$ is in $I$ for some $I \in \mathcal{I}$. Let $I=\left(x_{0}, x_{1}\right)_{B_{T}}$. We have $x_{1} \prec x$ (or $\left.x \prec x_{0}\right)$ and $x_{1} \in \operatorname{cl}_{B_{T}}\left(B_{T}\left\lceil\alpha^{+}\right)\left(\right.\right.$or $x_{0} \in \operatorname{cl}_{B_{T}}\left(B_{T}\left\lceil\alpha^{+}\right)\right.$). Let $u_{0}^{\prime}=x_{1}$ (or $u_{1}^{\prime}=x_{0}$ ). Then $u_{0}^{\prime}$ (or $\left.u_{1}^{\prime}\right) \in \operatorname{cl}_{B_{T}}\left(B_{T}\left\lceil\alpha^{+}\right)\right.$and $u_{0} \prec u_{0}^{\prime} \prec x$ (or $\left.x \prec u_{1}^{\prime} \prec u_{1}\right)$. So we always can choose $u_{0}^{\prime}, u_{1}^{\prime} \in \operatorname{cl}_{B_{T}}\left(B_{T}\left\lceil\alpha^{+}\right)\right.$such that $x \in\left(u_{0}^{\prime}, u_{1}^{\prime}\right)_{B_{T}} \subset U$. Since $\mathcal{C}$ is the base of $\mathrm{cl}_{B_{T}}\left(B_{T}\left\lceil\alpha^{+}\right)\right.$, there is a $C \in \mathcal{C}$ such that $x \in C \subset\left(u_{0}^{\prime}, u_{1}^{\prime}\right)_{B_{T}} \cap \operatorname{cl}_{B_{T}}\left(B_{T}\left\lceil\alpha^{+}\right)\right.$. It follows that $x \in J_{C} \subset\left(u_{0}^{\prime}, u_{1}^{\prime}\right)_{B_{T}} \subset U$. Thus (3) is true.

$(3) \Rightarrow(4) \Rightarrow(1)$ is obvious.

Lemma 3. Let $X$ be a subspace of $\left\langle B_{T}, \lambda, \preccurlyeq\right\rangle$. If $X$ is not separable, then there is a collection of disjoint open subsets of $X$ of the cardinal $\omega_{1}$.

Proof. Let $X_{1}=\left(B_{T 1} \cup B_{T 0}\right) \cap X, X_{0}=X-X_{1}$ and let

$$
T^{\prime}=\bigcup\left\{x \mid x \in X_{0}\right\} \cup\left(\bigcup\left\{x\left\lceil\eta_{x} \mid x \in X_{1}\right\}\right) .\right.
$$

If $\left|X_{1}\right|>\omega_{0}$, then the set $\left\{\eta_{x} \mid x \in X_{1}\right\}$ is cofinal in $\omega_{1}$ since each $T_{\alpha}$ is countable. If $\left|X_{1}\right| \leqslant \omega_{0}$, then $X_{0}$ is not separable. In any case, it follows from Lemma 2 that ht $T^{\prime}=\omega_{1}$. So $T^{\prime}$ is also a special Aronszajn tree. Hence $T^{\prime}=\bigcup\left\{A_{n}^{\prime} \mid n \in \omega_{0}\right\}$, where $A_{n}^{\prime}=T^{\prime} \cap A_{n}$. Then there is an $n_{0} \in \omega_{0}$ such that $\left|A_{n_{0}}^{\prime}\right|=\omega_{1}$ since $\left|T^{\prime}\right|=\omega_{1}$. Suppose that $t \in A_{n_{0}}^{\prime}$. Then there is an $x \in X$ such that $t \in x$. By the definition of $T^{\prime}, x$ is not an endpoint of $B_{t}$. Hence $x \in \operatorname{int}_{B_{T}} B_{t} \cap X$. Since $A_{n_{0}}^{\prime}$ is an antichain of $T,\left\{\operatorname{int}_{B_{T}} B_{t} \cap X \mid t \in A_{n_{0}}^{\prime}\right\}$ is a collection of disjoint open sets of $X$ with the cardinal $\omega_{1}$. 


\section{THEOREM}

Theorem 4. $\left\langle B_{T}, \lambda, \preccurlyeq\right\rangle$ is a non-metrizable compact LOTS such that every subspace has a $\sigma$-minimal base for its relative topology.

Proof. As was mentioned at the begining of Section $2, B_{T}$ is compact. By Lemma $2, B_{T}$ is not separable. Therefore $B_{T}$ is not metrizable. We only need to show that every subspace of $B_{T}$ has a $\sigma$-minimal base for its relative topology. Let $X$ be a subspace of $B_{T}$. If $X$ is separable, then by Lemma $2, X$ has a countable base which clearly is a $\sigma$-minimal base.

In the following we assume that $X$ is not separable. By Lemma $2,\{\operatorname{bht}(x) \mid x \in$ $X\}$ is cofinal in $\omega_{1}$. Put

$$
T(X)=\bigcup\{x \mid x \in X\} .
$$

Then $T(X)$ is also a special Aronszajn tree since

$$
T(X)=\bigcup\left\{A_{n}(X) \mid n \in \omega_{0}\right\},
$$

where $A_{n}(X)=A_{n} \cap T(X)$.

It is obvious that $\left\{B_{t} \cap X \mid t \in A_{n}(X)\right\}$ is a disjoint collection. Hence $\left\{\operatorname{int}_{X}\left(B_{t} \cap\right.\right.$ $\left.X) \mid t \in A_{n}(X)\right\}$ is a disjoint collection of open sets in $X$, and so it is trivially minimal. Thus the collection $\left\{\operatorname{int}_{X}\left(B_{t} \cap X\right) \mid t \in T(X)\right\}$ is a $\sigma$-minimal collection of open sets in $X$. Of course, it is probably not a base for $X$ in general. But for quite large subsets of $X$, the collection serves as a base. In the rest of the proof, we will "refine" the collection $\left\{B_{t} \cap X \mid t \in T(X)\right\}$ to produce a base of $X$ keeping the $\sigma$-minimality. For this purpose, put

$$
\begin{aligned}
& E=B_{T 2} \cap X, \\
& D_{0}=B_{T 0}^{s u c 1} \cap X, \\
& D_{1}=\left(B_{T 1}^{s u c} \cup\left(B_{T 0}^{s u c}-B_{T 0}^{s u c 1}\right)\right) \cap X=\left(\left(B_{T 1}^{s u c} \cup B_{T 0}^{s u c}\right) \cap X\right)-D_{0}, \\
& G_{1}=B_{T 1}^{l i m} \cap X, \text { and } \\
& G_{0}=B_{T 0}^{l i m} \cap X .
\end{aligned}
$$

Then $X=\left(B_{T 1} \cup B_{T 0} \cup B_{T 2}\right) \cap X=D_{0} \cup D_{1} \cup E \cup G_{0} \cup G_{1}$.

In fact, for every point in $E$ and $D_{1},\left\{\operatorname{int}_{X}\left(B_{t} \cap X\right) \mid t \in T(X)\right\}$ contains a neighborhood base of the point (see Cases 1 and 3 below). Now we consider the points in $D_{0}, G_{0}$ and $G_{1}$. Suppose that $t \in T(X)$. By Fact 4 , we may write $B_{t}$ as $\left[b_{0}(t), b_{1}(t)\right]_{B_{T}}$.

(i) If there is an $x \in D_{0}$ such that $t \in x \cap T_{\alpha}$ and $\eta_{x}=\alpha^{+}$, then $x \in \operatorname{int}_{B_{T}} B_{t}$. Let $\mathcal{H}(t)=\left\{H(t, k) \mid k \in \omega_{0}\right\}$ be a countable neighborhood base at $x$ in $X$ such that each $H(t, k) \subset B_{t}$.

(ii) Let $s_{1}(t)$ be the minimum point in $B_{t}$ such that $\left[s_{1}(t), b_{1}(t)\right]_{B_{T}} \cap G_{1}$ is separable. Because of the compactness and first countability of $B_{T}, s_{1}(t)$ exists. For $\left[s_{1}(t), b_{1}(t)\right)_{B_{T}} \cap G_{1}$, if it is not empty, by Lemma 2, there is a countable collection $\mathcal{S}_{1}^{r}(t)=\left\{S^{r}(t, k) \mid k \in \omega_{0}\right\}$ in $X$ which contains neighborhood bases of all points in $\left[s_{1}(t), b_{1}(t)\right)_{B_{T}} \cap G_{1}$ since it is separable. Clearly we may assume that each $S^{r}(t, k) \subset B_{t}$.

If $\left(b_{0}(t), s_{1}(t)\right)_{B_{T}} \cap G_{1}$ is not empty, then it is not separable. By Lemma $1, B_{T}$ is first countable, so we may take an increasing sequence $\left\{d_{k}(t)\right\}$ in $\left[b_{0}(t), s_{1}(t)\right)_{B_{T}}$ 
such that $\left\{d_{k}(t)\right\}$ converges to $s_{1}(t)$. Also there is a $k \in \omega_{0}$ such that $\left[b_{0}(t), d_{k}(t)\right]_{B_{T}}$ $\cap G_{1}$ is not separable, hence we may assume that $b_{0}(t) \prec d_{0}(t)$ and $\left(b_{0}(t), d_{0}(t)\right)_{B_{T}}$ $\cap G_{1}$ is not separable. Let $G_{1}(t, k)=\left(b_{0}(t), d_{k}(t)\right)_{B_{T}} \cap G_{1}$. For each $x \in G_{1}(t, k)$, let $\left\{g_{1}(t, k, x, j) \mid j \in \omega_{0}\right\}$ be an increasing sequence in $\left(b_{0}(t), d_{k}(t)\right)_{B_{T}}$ converging to $x$. Let

$$
K_{1}(t, k)=\left\{g_{1}(t, k, x, j) \mid j \in \omega_{0}, x \in G_{1}(t, k)\right\} .
$$

Then $\left|K_{1}(t, k)\right|=\omega_{1}$. Observe that the separability is a hereditary property in GO-spaces. Since $\left(d_{k}(t), s_{1}(t)\right)_{B_{T}} \cap G_{1}$ is not separable by the minimality of $s_{1}(t)$, so is $\left(d_{k}(t), s_{1}(t)\right)_{B_{T}} \cap X$. By Lemma 3 , there is a collection $\mathcal{O}_{1}(t, k)$ of disjoint open sets in $X$ contained in $\left(d_{k}(t), s_{1}(t)\right)_{B_{T}}$ such that $\left|\mathcal{O}_{1}(t, k)\right|=\omega_{1}$. Let

$$
\phi_{1, t, k}: K_{1}(t, k) \rightarrow \mathcal{O}_{1}(t, k)
$$

be a bijection. Put

$$
\begin{aligned}
& \mathcal{G}_{1}(t, k)= \\
&\left\{\left(\left(g_{1}(t, k, x, j), d_{k}(t)\right)_{B_{T}} \cap X\right) \cup \phi_{1, t, k}\left(g_{1}(t, k, x, j)\right) \mid\right. \\
&\left.\quad j \in \omega_{0}, x \in G_{1}(t, k)\right\} .
\end{aligned}
$$

Notice that $\phi_{1, t, k}\left(g_{1}(t, k, x, j)\right) \subset\left(d_{k}(t), s_{1}(t)\right)_{B_{T}}$ and $g_{1}(t, k, x, j) \in K_{1}(t, k) \subset$ $\left[b_{0}(t), d_{k}(t)\right)_{B_{T}}$. Since $\mathcal{O}_{1}(t, k)$ is a disjoint collection, it follows that $\mathcal{G}_{1}(t, k)$ is a minimal collection of open sets in $X$ contained in $B_{t}$.

(iii) Similar to (ii), for $G_{0}$, we may define $s_{0}(t),\left\{c_{k}(t)\right\},\left\{g_{0}(t, k, x, j) \mid j \in \omega_{0}\right\}$, $\phi_{0, t, k}, \mathcal{G}_{0}(t, k)$, and $\left\{S^{l}(t, k) \mid k \in \omega_{0}\right\}$ corresponding to $s_{1}(t),\left\{d_{k}(t)\right\},\left\{g_{1}(t, k, x, j) \mid\right.$ $\left.j \in \omega_{0}\right\}, \phi_{1, t, k}, \mathcal{G}_{1}(t, k)$, and $\left\{S^{r}(t, k) \mid k \in \omega_{0}\right\}$ in (ii) respectively by replacing minimum and increasing by maximum and decreasing respectively.

Thus each of the following collections, if it is defined for $t$, is a minimal collection of open sets in $X$ contained in $B_{t}$.

(1) $\left\{\operatorname{int}_{X}\left(B_{t} \cap X\right)\right\}$;

(2) $\{H(t, k)\}$ for $k \in \omega_{0}$;

(3) $\mathcal{G}_{0}(t, k)$ for $k \in \omega_{0}$;

(4) $\mathcal{G}_{1}(t, k)$ for $k \in \omega_{0}$;

(5) $\left\{S^{r}(t, k)\right\}$ for $k \in \omega_{0}$;

(6) $\left\{S^{l}(t, k)\right\}$ for $k \in \omega_{0}$.

For each $t \in T(X)$, enumerate all these minimal collections in (1) to (6) above which are defined for $t$ as $\left\{\mathcal{B}(t, k) \mid k \in \omega_{0}\right\}$.

For $t_{1}, t_{2} \in A_{n}(X), n \in \omega_{0}$, if $t_{1} \neq t_{2}$, then $B_{t_{1}} \cap B_{t_{2}}=\emptyset$ since $A_{n}(X)$ is an antichain of $T(X)$. Each element of $\mathcal{B}(t, k)$ is a subset of $B_{t}$. It follows that the collection $\mathcal{B}_{k, n}=\bigcup\left\{\mathcal{B}(t, k) \mid t \in A_{n}(X)\right\}$ is also a minimal collection for each pair $k, n \in \omega_{0}$.

There are three special points we should consider if they are in $X: z_{0} \in D_{0}$ satisfying $\eta_{z_{0}}=0$, the maximum point $z_{1}$ of $B_{T}$, and the minimum point $z_{2}$ of $B_{T}$. Let $\mathcal{W}\left(z_{i}\right)=\left\{W_{i, n} \mid n \in \omega_{0}\right\}$ be a countable neighborhood base at $z_{i}$ in $B_{T}$ for $i=0,1,2$ respectively. Hence

$$
\mathcal{B}=\bigcup\left\{\mathcal{B}_{k, n} \mid k, n \in \omega_{0}\right\} \cup\left\{W_{i, n} \cap X \mid n \in \omega_{0}, i=0,1,2\right\}
$$

is a $\sigma$-minimal collection in $X$. 
Now we prove that $\mathcal{B}$ is a base of $X$. Trivially $\mathcal{B}$ contains the neighborhood bases at $z_{i}$ for $i=0,1,2$. So suppose that $x \in X$ with $x \neq z_{i}$ where $i=0,1,2$ and $U$ is a neighborhood of $x$ in $X$. Then there is an open interval $(a, b)_{B_{T}}$ of $B_{T}$ such that $x \in(a, b)_{B_{T}} \cap X \subset U$. Then by the definition of the ordering on $B_{T}$,

$$
a_{N_{a \cap x}}<_{N_{a \cap x}} x_{N_{a \cap x}} \text { and } x_{N_{x \cap b}}<_{N_{x \cap b}} b_{N_{x \cap b}} .
$$

Case 1. $x \in E$. Let

$$
\alpha=\max \{\operatorname{ht}(a \cap x), \operatorname{ht}(x \cap b)\} \text { and }\{t\}=x \cap N\left(x, \alpha^{+}\right) .
$$

Then $t \in T(X)$. It follows from $(*)$ that $B_{t} \subset(a, b)_{B_{T}}$ since for any $y \in B_{t}, a \cap y=$ $a \cap x, x \cap b=y \cap b$, and $y_{N_{a \cap y}}=x_{N_{a \cap x}}, y_{N_{y \cap b}}=x_{N_{x \cap b}}$. It is obvious that $x \in B_{t}$. Moreover by Fact $4, x$ cannot be the maximum point or minimum point of $B_{t}$ since $x \in E$. Hence $x \in \operatorname{int}_{X}\left(B_{t} \cap X\right) \subset(a, b)_{B_{T}} \cap X \subset U$ and $\operatorname{int}_{X}\left(B_{t} \cap X\right) \in \mathcal{B}$.

Case 2. $x \in D_{0}$. Since $x \neq z_{0}, \eta_{x}=\alpha^{+}$for some $\alpha$. Let $\{t\}=x \cap T_{\alpha}$. It follows from (i) that for some $k_{0} \in \omega_{0}, x \in H\left(t, k_{0}\right) \subset U$ and $H\left(t, k_{0}\right) \in \mathcal{B}$.

Case 3. $x \in D_{1}$. If $x \in B_{T 1}^{\text {suc }}$, since $x$ is not the maximum point of $B_{T}, x \cap$ $N\left(x, \eta_{x}\right) \neq\left\{a\left(N\left(x, \eta_{x}\right)\right)_{\omega_{0}}\right\}$ even if $\eta_{x}=0$. So there is a $y \in B_{T 0}^{s u c}$ such that $\eta_{y}=\eta_{x}, \quad N\left(y, \eta_{y}\right)=N\left(x, \eta_{x}\right)$ and if $x \cap N\left(x, \eta_{x}\right)=\left\{a\left(N\left(x, \eta_{x}\right)\right)_{i}\right\}$, then $y \cap$ $N\left(x, \eta_{x}\right)=\left\{a\left(N\left(x, \eta_{x}\right)\right)_{i+1}\right\}$, where $i \in \omega_{0}$. It is easy to check that $y$ is an immediate successor of $x$ in $B_{T}$. Remember $x \in(a, b)_{B_{T}}$. Let

$$
\alpha=\max \left\{\operatorname{ht}(a \cap x), \eta_{x}\right\} \text { and }\{t\}=x \cap N\left(x, \alpha^{+}\right) .
$$

Then $x$ is the maximum point of $B_{t}$ and $x \in \operatorname{int}_{X}\left(B_{t} \cap X\right)$ since $x$ has an immediate successor in $B_{T}$. Since for any $y \in B_{t}, a \cap y=a \cap x$ and $y_{N_{a \cap y}}=x_{N_{a \cap x}}$, by (*), we have $x \in \operatorname{int}_{X}\left(B_{t} \cap X\right) \subset(a, b)_{B_{T}} \cap X \subset U$ and $\operatorname{int}_{X}\left(B_{t} \cap X\right) \in \mathcal{B}$. Similarly, if $x \in B_{T 0}^{\text {suc }}$, then $x$ has an immediate predecessor in $B_{T}$ since $x \notin D_{0}$. It follows that for some $t \in T(X), x \in \operatorname{int}_{X}\left(B_{t} \cap X\right) \subset(a, b)_{B_{T}} \cap X \subset U$ and $\operatorname{int}_{X}\left(B_{t} \cap X\right) \in \mathcal{B}$.

Case 4. $x \in G_{1}$. If ht $(a \cap x)<\eta_{x}$, let $\alpha=\max \{\operatorname{ht}(a \cap x)$, ht $(x \cap b)\}$. Then $\alpha^{+}<\eta_{x}$ since $x \in B_{T 1}^{\lim }$. Let $\{t\}=x \cap N\left(x, \alpha^{+}\right)$. Similar to Case $1, x \in \operatorname{int}_{X}\left(B_{t} \cap X\right) \subset U$ and $\operatorname{int}_{X}\left(B_{t} \cap X\right) \in \mathcal{B}$.

If ht $(a \cap x)>\eta_{x}$, let $\alpha=\operatorname{ht}(x \cap b)$. Then $\alpha^{+}<\eta_{x}$. Let $\{t\}=x \cap N\left(x, \alpha^{+}\right)$. Then $x \in\left(b_{0}(t), b_{1}(t)\right)_{B_{T}}$ and for any $y \in B_{t}, y \prec b$. If $s_{1}(t) \preccurlyeq x$, by (ii), there is some $k_{0} \in \omega_{0}$ such that $x \in S^{r}\left(t, k_{0}\right) \subset U$ and $S^{r}\left(t, k_{0}\right) \in \mathcal{B}$. If $x \prec s_{1}(t)$, let $\left\{d_{k}(t)\right\},\left\{g_{1}(t, k, x, j) \mid j \in \omega_{0}\right\}$ and $\mathcal{O}_{1}(t, k)$ be the sequences and collection in (ii). Since $x \prec s_{1}(t)$, there is a $k_{0} \in \omega_{0}$ such that $x \prec d_{k_{0}} \prec s_{1}(t)$ and there is some $j_{0} \in \omega_{0}$ such that $a \prec g_{1}\left(t, k_{0}, x, j_{0}\right) \prec x$. Let

$$
V=\left(\left(g_{1}\left(t, k_{0}, x, j_{0}\right), d_{k_{0}}\right)_{B_{T}} \cap X\right) \cup \phi_{1, t, k_{0}}\left(g_{1}\left(t, k_{0}, x, j_{0}\right)\right) .
$$

Then $x \in V$ and $V \subset(a, b)_{B_{T}} \cap X \subset U$ and $V \in \mathcal{B}$.

Case 5. $x \in G_{0}$. Similar to Case 4 , we can choose a $V \in \mathcal{B}$ such that $x \in V \subset U$.

Thus we have proved that $\mathcal{B}$ is a $\sigma$-minimal base of $X$, and this completes the proof.

Remark 1. Suppose that $X$ is a compact LOTS. Insert a copy of the usual interval $(0,1)$ of real line into each jump $(A, B)$ in $X$. Then the resulting LOTS $X^{\prime}$ will be a continuum. If $X$ is not metrizable and satisfies the conditions in Problem 1, so does $X^{\prime}$ since $X^{\prime}-X$ is a union of disjoint open intervals in $X^{\prime}$ and each of those open 
intervals is homeomorphic to $(0,1)$. Then it is easy to check that $X^{\prime}$ satisfies first countability, non-separability and that the closure of any countable subset of $X^{\prime}$ is second countable. This means that $X^{\prime}$ is an Aronszajn continuum. By Theorem 4, we may claim that there is an Aronszajn continuum with the interval topology which is a counterexample for Problem 1 and that any counterexample for Problem 1 must be a subspace of an Aronszajn continuum with the interval topology.

Remark 2. Since a compact quasi-developable space is metrizable, Theorem 4 also negatively answers the problem about whether a LOTS which has $\sigma$-minimal bases hereditarily is quasi-developable (see [6]).

\section{ACKNOWLEDGMENT}

The author would like to thank Professor Takao Hoshina for his guidance. Thanks are also due to Doctor Kazuhiro Kawamura and the referee for their helpful comments.

\section{REFERENCES}

[1] C. E. Aull, Quasi-development and $\delta \theta$-base, J. London Math. Soc. (2), 9(1974), 197-204. MR 52:9171

[2] H. R. Bennett and E. S. Berney, Space with $\sigma$-minimal base, Topology Proceedings 2(1977), 1-10. MR 80k:54050

[3] H. R. Bennett and D. J. Lutzer, Ordered space with $\sigma$-minimal base, Topology Proceedings 2(1977), 371-382. MR 80j:54027

[4] _ Problems in Perfect Ordered Spaces, in: Open Problems in Topology, J. van Mill and G. M. Reed ed. (North-Holland, Amsterdam, 1990). CMP 91:03

[5] - A metric space of A. H. Stone and an example concerning $\sigma$-minimal bases, Proc. Amer. Math. Soc. 126 (1998), 2191-2196. MR 98j:54054

[6] _ Metrization, quasi-developments and $\sigma$-minimal bases, Q. and A. in Gen. Top. 2(1984), 73-76. CMP 17:08

[7] R. Engleking, General Topology, (Hedermann, Berlin, 1989). MR 91c:54001

[8] I. Juhász, Cardinal Functions in Topology, (MC Tract 34, Mathematical Centre, Amsterdam, 1975). CMP 98:10

[9] D. J. Lutzer, Twenty questions on ordered spaces, in: Topology and Order Structures (Part 2), H. R. Bennett and D. J. Lutzer, editors. (MC Tract 169, Mathematical Centre, Amsterdam, 1983). MR 85h:54058

[10] S. Todorčević, Trees and linearly ordered sets, in: Handbook of Set-Theoretic Topology, K. Kunen and J. E. Vanghan editors, (North-Holland, Amsterdam. 1984). MR 86h:54040

Department of Mathematics, Changchun Teachers College, Changchun 130032, China Current address: Institute of Mathematics, University of Tsukuba, Tsukuba-shi, Ibaraki 305, Japan

E-mail address: shi@abel.math.tsukuba.ac.jp 